\title{
Escalamiento de antimicrobianos con la inclusión de una lista de verificación en la unidad de cuidados intensivos pediátricos
}

\author{
Antibiotic escalation with the inclusion of a checklist in the pediatric intensive care unit
}

\author{
Mirta N. Mesquita ${ }^{1,2}$, Laura E. Godoy ${ }^{1,3}$, Hayat A. Kabboutt ${ }^{1}$ y María L. Servin ${ }^{1}$
}

${ }^{1}$ Hospital General Pediátrico Niños de Acosta Ñu. San Lorenzo, Paraguay.
${ }^{2}$ Universidad Católica Nuestra Señora de la Asunción. Asunción, Paraguay.
${ }^{3}$ Facultad en Ciencias de la Salud de la Universidad del Pacifico. Asunción, Paraguay.

Financiamiento: No hubo financiamiento externo

Conflictos de interés: ninguno.

Recibido (segunda versión): 10 de octubre de 2019 / Aceptado: 8 de julio de 2020

\section{Resumen}

Introducción: La vigilancia de la administración de los antimicrobianos mejora su prescripción. Objetivo: Disminuir el escalamiento en el uso de antimicrobianos a las $48 \mathrm{~h}$ del inicio, en la unidad de cuidados intensivos pediátricos (UCIP). Material y Métodos: Estudio de cohortes ambispectivo, realizado en una UCIP e incluyó pacientes ingresados que recibieron antibioterapia, implementando una lista de verificación del uso de antimicrobianos (checklist) en forma prospectiva, Se comparó con una cohorte histórica previa al checklist. Fueron evaluados el escalamiento de antimicrobianos a las $48 \mathrm{~h}$, la consulta con infectólogos y la indicación de vancomicina .La comparación de las variables entre las dos cohortes se realizó con la prueba de $\chi^{2}$, el test de Fischer y la U de Mann Whitney. Los resultados principales se expresaron en RR y RAR. Estudio aprobado por el Comité de Ética institucional. Resultados: Ingresaron 70 pacientes en la etapa del checklist, y fueron comparados con 124 pacientes de la cohorte histórica. La introducción del checklist redujo el escalamiento de antimicrobianos a las $48 \mathrm{~h}$, desde 56,4 a 21,4\% (p < 0,0001) con una RAR de $35 \%$; la prescripción de la vancomicina desde 64,5 a $40 \%(\mathrm{p}<0,001)$, RAR $24,5 \%$ e incrementó la consulta con infectólogo en $23 \%$, (desde 9,6 a $32,8 \%)(\mathrm{p}<0,0001)$. No hubo diferencias en la mortalidad y duración de la antibioterapia a los 10 días. Conclusiones: El checklist permitió disminuir el escalamiento de antimicrobianos a las $48 \mathrm{~h}$ del inicio, la indicación de vancomicina y aumentó la consulta con infectólogos.

Palabras clave: cuidados críticos; pediatría; antimicrobianos; uso apropiado; lista de verificación; vigilancia.

\section{Abstract}

Background: Antibiotic surveillance improves the appropriate antibiotic therapy. Aim: To decrease the antibiotic scaling, 48 hours after starting prescription in the pediatric intensive care unit (PICU). Methods: A ambispective cohort study was performed in the PICU including patients admitted in whom antibiotic therapy was started and a checklist was applied prospectively. They were compared with a historical cohort, prior the checklist. The main outcome was the antibiotic scaling 48 hours after starting and the secondary endpoints were consultation with infectious diseases (ID) specialist and vancomycin prescription. To compare the variables between the two cohorts, the $\chi^{2}$ test, Fischer test and U Mann-Whitney test were used. The results of the main variables were expressed in RR and RAR. The study was approved by the institution's Ethics Committee. Results: 70 patients were admitted in the checklist cohort and they were compared with 124 patients of the historical cohort. The checklist implementation decreased the antibiotic scaling at $48 \mathrm{~h}$ after starting from 56.4 to $21.4 \%(\mathrm{p}<0.0001) \mathrm{ARR}=35 \%$ and vancomycin prescription from 64.5 to $40 \%(\mathrm{p}<0.001)$ ARR $=24.5 \%$. The consultation with ID specialist increased from 9.6 to $32.8 \%(\mathrm{p}<0.0001)$. There were no differences in mortality and duration of antibiotic therapy at 10 days of hospitalization. Conclusion: The checklist implementation decreased the antibiotic scaling, $48 \mathrm{hs}$ after starting and the vancomycin prescription while the ID specialist consultation increased.

Keywords: critical care unit; pediatrics; antibiotic; stewardship; checklist; surveillance. 


\section{Introducción}

L as infecciones graves constituyen un motivo de ingreso frecuente a la unidad de cuidados intensivos pediátricos (UCIP). La prescripción de más de un antimicrobiano, es muy elevada en esta población $\mathrm{y}$ es una de las medicaciones que se prescriben con mayor frecuencia en las unidades de cuidados intensivos ${ }^{1,2}$.

Las decisiones sobre el tipo de antimicrobiano a utilizar, así como el tiempo de duración del tratamiento, son muy complejas en la UCIP. Aunque la indicación precoz de antibioterapia es crucial para la sobrevida del paciente, la decisión de suspensión muchas veces es un desafío para el médico ${ }^{3}$. La utilización precoz de bio-marcadores específicos podrían ayudar en la identificación de aquellos pacientes en quienes se podría suspender el tratamiento antimicrobiano en forma segura ${ }^{4}$.

El uso apropiado de los antimicrobianos implica, realizar el diagnostico correctamente, tomar los cultivos correspondientes, utilizar la antibioterapia adecuada precozmente, prescribir la dosis correctamente, realizar el seguimiento de la evolución clínica del paciente de modo a disminuir el escalamiento de los antimicrobianos y confirmar la sensibilidad del microorganismo ${ }^{5}$. Sin embargo, a pesar de las guías de prescripción, frecuentemente se los utiliza en forma inapropiada, en especial en países en vías de desarrollo. La consecuencia principal es la emergencia de microorganismos multi-resistentes responsables de infecciones graves, que incrementan la morbi-mortalidad y los costos de los cuidados de la salud ${ }^{6,7}$.

La creación de programas de administración de antimicrobianos ha mejorado la prescripción, reduciendo el uso inapropiado y la utilización de antimicrobianos de amplio espectro ${ }^{8}$. Sin embargo, la implementación de estas herramientas no se ha generalizado debido a la falta de financiamiento, recursos humanos y concienciación, tanto del personal médico como de los responsables de las políticas de salud pública, principalmente en países en vías de desarrollo?.

El hospital pediátrico donde se desarrolló el estudio, como todos los hospitales del país, no cuenta con un programa de administración de antimicrobianos. El objetivo del presente estudio fue determinar si la implementación de una lista de verificación del uso de antimicrobianos (check list) en la UCIP, basado en el modelo utilizado por Stocker y cols. ${ }^{10}$, disminuye el escalamiento de antimicrobianos, las $48 \mathrm{~h}$ de haberse iniciado y aumenta la interconsulta con los especialistas en infectología.

\section{Material y Métodos}

\section{Diseño y lugar del estudio}

Estudio de cohortes, ambispectivo, realizado en la UCIP de un hospital pediátrico universitario de tercer nivel de referencia, que recibe pacientes críticos remitidos de otros hospitales de todo el país. Se incluyó:

- una cohorte prospectiva de pacientes ingresados en la UCIP desde 1 de octubre del 2016, fecha en que se implementó una lista de verificación de antimicrobianos en todos los pacientes que recibían estos fármacos, hasta 30 de enero de 2017 (etapa check list);

- una cohorte histórica de comparación, previa a la implementación del check list en el período de 1 de enero al 30 de junio de 2015.

\section{Cohorte prospectiva}

Selección de pacientes e implementación del check list: Fueron elegibles los pacientes ingresados a la UCIP que recibían antimicrobianos y contaban con el consentimiento informado de los padres o encargados. Se realizó un muestreo no probabilístico de casos consecutivos. Al ingreso a la UCIP, a la historia clínica de los pacientes se adjuntó una lista de verificación de antimicrobianos, que los médicos de la sala debían llenar diariamente durante las primeras $48 \mathrm{~h}$ de iniciado el antimicrobiano, y a los 10 días de hospitalización si aún se encontraban en sala. Si el alta, traslado o fallecimiento tenía lugar antes de los 10 días, se anotaba los días de hospitalización y días totales de antibioterapia, pero si ocurría antes de las $48 \mathrm{~h}$ de ingreso se los excluía del estudio. El check list (ver Anexo) contenía datos demográficos del paciente, el motivo de inicio de antimicrobiano (sospecha de infección o profilaxis), procedimientos invasivos en el momento de la prescripción, (asistencia respiratoria mecánica, nutrición parenteral y catéter venoso central), signos clínicos de infección, toma de cultivos (sangre, líquido cefalorraquídeo, orina y liquido pleural), tratamiento empírico inicial. A las $48 \mathrm{~h}$ se evaluaba a los pacientes con los resultados de los cultivos, justificando la conducta asumida con respecto a los antimicrobianos (suspender, seguir igual esquema o cambiar), los tipos de antimicrobianos utilizados y la interconsulta con infectologos.A los 10 días del ingreso se verificaba, el estado del paciente, (vivo, dado de alta o fallecido) y al abandonar la UCIP los días totales de antibioterapia.(ver Anexos) Antes de iniciar el estudio, los médicos del staff de la UCIP fueron capacitados sobre el llenado del check list. Una de las investigadoras coordinó el reclutamiento y el llenado correcto de la ficha.

El primer punto a evaluar fue el escalamiento del antimicrobiano definido como el cambio a otro de mayor espectro a las $48 \mathrm{~h}$ de iniciado el tratamiento. Los puntos de evaluación secundarios fueron la frecuencia de prescripción de vancomicina y la frecuencia de interconsulta con infectólogos.

\section{Tamaño de la muestra}

Para el cálculo del tamaño de la muestra se planteó la hipótesis de disminuir en $50 \%$ el porcentaje de esca- 
lamiento de antimicrobianos a las $48 \mathrm{~h}$, sin justificación. Utilizando la prueba $\chi^{2}$, para un alfa bilateral de 0,05 y una potencia de $80 \%$ fue necesario incluir 68 pacientes. El Comité de Ética de la institución aprobó el protocolo $\mathrm{y}$ el formulario de consentimiento informado escrito.

\section{Cohorte histórica}

En este período se realizó la revisión de historias clínicas de los pacientes ingresados en el período previo a la implementación del check list. Los criterios de inclusión y exclusión de los pacientes, las variables recogidas, así como los puntos de corte primario y secundario, fueron los mismos que en el período de check list. El tamaño de la muestra de la cohorte histórica se estimó en 120 participantes, considerando que la proporción esperada de uso de antimicrobianos en la UCIP fue de $90 \%$, con una precisión de 3\% y un intervalo de confianza de 95\% para poblaciones finitas.

\section{Análisis de los datos}

Los datos se analizaron con SPSS v21. Las variables cualitativas se expresaron en porcentajes y las cuantitativas en medianas con sus valores mínimo y máximo. La comparación de las variables en las dos cohortes se realizó utilizando la tabla de contingencia, la prueba de $\chi^{2}$ de Pearson y el test de Fisher según estaba indicado, para las variables cualitativas La relación entre variables cuantitativas se realizó por la U de Mann Whitney. La disminución del escalamiento se expresó como RR y RRA (reducción del riesgo absoluto). Se consideró un valor alfa inferior al 5\%.

\section{Resultados}

En la etapa prospectiva fueron incluidos 70 pacientes. Las características demográficas, procedimientos invasivos y principales signos de infección fueron comparados con 124 pacientes de la cohorte histórica. En la Tabla 1 se observan los datos en ambas cohortes.

Se analizaron el motivo de prescripción de antimicrobianos y los tipos de cultivos realizados, sin observarse diferencias significativas entre ambas cohortes (Tabla 2).

\begin{tabular}{|c|c|c|c|}
\hline Variables & Cohorte histórica ( $n: 124)$ & Etapa check list (n: 70) & p \\
\hline Edad (mediana) (min-max) & 10 meses $(1-204)$ & 8,5 meses $(1-204)$ & $0,9^{1}$ \\
\hline Género masculino. N (\%) & $68(54,8)$ & $44(62,9)$ & $0,3^{2}$ \\
\hline \multicolumn{4}{|l|}{ Procedimientos invasivos. N (\%) } \\
\hline ARM & $96(77,4)$ & $53(75,7)$ & $0,92^{2} \quad 0,97^{3}$ \\
\hline CVC & $56(45,2)$ & $31(44,2)$ & $0,97^{2}$ \\
\hline NPT & $9(7,3)$ & $5(7,1)$ & $0,79^{2}$ \\
\hline \multicolumn{4}{|l|}{ Signos de infección*. N (\%) } \\
\hline Respiratorio (distress respiratorio, rx de tórax anormal) & $50(40,3)$ & $30(42,8)$ & $0,84^{2}$ \\
\hline Alteración del recuento de leucocitos & $36(29)$ & $18(25,7)$ & $0,74^{2}$ \\
\hline Distermia & $25(20)$ & $15(21,4)$ & $0,90^{2}$ \\
\hline Inestabilidad hemodinámica & $25(20)$ & $12(17)$ & $0,74^{2}$ \\
\hline
\end{tabular}

Tabla 2. Motivo de inicio de antimicrobianos y obtención de cultivos en ambas etapas del estudio

\begin{tabular}{|c|c|c|c|}
\hline & $\begin{array}{c}\text { Cohorte histórica }(n=124) \\
N \quad(\%)\end{array}$ & $\begin{array}{c}\text { Etapa check list }(\mathrm{n}=70) \\
\mathrm{N}(\%)\end{array}$ & p \\
\hline \multicolumn{4}{|l|}{ Motivo inicio TAM } \\
\hline Sospecha de infección (tratamiento empírico inicial) & $114(92)$ & $63(90)$ & $0,85^{1}$ \\
\hline Profilaxis & $10(8)$ & $7(10)$ & $0,84^{1}$ \\
\hline \multicolumn{4}{|l|}{ Obtención de cultivos } \\
\hline Policultivos (sangre, LCR, orina, secreciones) & $99(79,8)$ & $60(85,7)$ & $0,40^{1}$ \\
\hline Sólo hemocultivo & $25(20,1)$ & $10(14,3)$ & $0,40^{1}$ \\
\hline
\end{tabular}


En relación al seguimiento a las $48 \mathrm{~h}$ de iniciado el tratamiento empírico, en base a la posibilidad de infección se consideró la suspensión, el cambio a otro antimicrobiano o continuar con el esquema inicial. Además, se recogió el dato sobre la interconsulta con los infectológos del hospital (Tabla 3).

El cambio de los antimicrobianos se consideró escalamiento porque en todos los casos se pasó a antimicrobianos de mayor espectro. Con la implementación del check list el escalamiento se redujo con un RR de 0,42 (IC 95\% 0,31-0,5) y una RAR de $35 \%$ y un NNT de 2,8 . El aumento de la consulta con infectólogos aumentó en forma significativa mediante el check list con un RR 0,29 (IC95\% 0,16-9,55).

La reducción del uso de vancomicina con el check list fue RR 0,59 (IC 95\%-0,44-0,80) y una RAR de 24,5\%.

Todos los pacientes en ambas etapas recibieron dos antimicrobianos como tratamiento empírico inicial, excepto los casos de profilaxis. La combinación más utilizada fue cefotaxima más vancomicina. Se recogieron los cinco antimicrobianos más utilizados en ambas etapas. La reducción del uso de vancomicina con el check list fue RR 0,59 (IC 95\%-0,44-0,80) y una RAR de 24,5\%. Se recogieron datos del estado de los pacientes a los 10 días de hospitalización así como los días totales de antimicrobianos hasta abandonar la UCIP (alta o fallecido) (Tabla 4).

Los hallazgos positivos de los hemocultivos tomados al ingreso, en la cohorte histórica, fueron: sobre 114 pacientes, 4 cultivos positivos $(3,5 \%)$, mientras que en la etapa check list fueron de $10 \%(7 / 70) \mathrm{P}=0,10$ (Test Fisher). Los microorganismos en la cohorte histórica fueron Staphyloccoccus aureus resistente a meticilina (2/4), Streptococcus pneumoniae (1/4) y Escherichia coli (1/4). En la etapa check list Escherichia coli (2/7), Neisseria meningitidis $(2 / 7), S$. aureus resistente a meticilina (1/7), Streptococcus grupo viridans (1/7) y Haemophilus influenzae (1/7).

\section{Discusión}

Tabla 3. Decisión tomada por los médicos sobre la antibioterapia a las $48 \mathrm{~h}$ de haberse iniciado el tratamiento empírico inicial, y frecuencia de interconsulta con infectólogos (n: 194)

\begin{tabular}{lccc}
\hline Evaluación a las $48 \mathbf{~}$ & $\begin{array}{c}\text { Cohorte histórica } \\
(\mathbf{n}=\mathbf{1 2 4}) \\
\mathbf{N ~}(\mathbf{\%})\end{array}$ & $\begin{array}{c}\text { Etapa check list } \\
(\mathbf{n}=\mathbf{7 0}) \\
\mathbf{N ~}(\%)\end{array}$ & $\mathbf{p}$ \\
\hline Cambio (escalamiento) & $70(56,4)$ & $15(21,4)$ & $<0,0001^{1}$ \\
Suspensión & $7(5,6)$ & $6(8,6)$ & $0,62^{2}$ \\
Sin cambios & $47(38)$ & $49(70)$ & $0,0001^{1}$ \\
Interconsulta con infectología & $12(9,6)$ & $23(32,8)$ & $0,0001^{1}$ \\
\hline${ }^{1} \chi^{2} .{ }^{2}$ Test de Fisher. & & & \\
\hline
\end{tabular}

Tabla 4. Los 5 antimicrobianos más utilizados, en la cohorte histórica y la indicación de los mismos en la etapa prospectiva con el check list. Se detallan también los días de antibioterapia y mortalidad a los 10 días de hospitalización en ambas fases del estudio

\begin{tabular}{lccc}
\hline $\begin{array}{l}\text { Antimicrobianos más utilizados } \\
\text { en la etapa prospectiva }\end{array}$ & $\begin{array}{c}\text { Cohorte histórica } \\
(\mathbf{n}=\mathbf{1 2 4}) \\
\mathbf{N ~ ( \% )}\end{array}$ & $\begin{array}{c}\text { Etapa check list } \\
(\mathbf{n}=\mathbf{7 0})\end{array}$ & $\mathbf{p}$ \\
\hline Nancomicina & $80(64,5)$ & $28(40)$ & $0,001^{1}$ \\
Cefotaxima & $67(54)$ & $39(55,7)$ & $0,9^{1}$ \\
\hline Ciprofloxacina & $47(37,9)$ & 0 & $0,0001^{2}$ \\
\hline Clindamicina & $36(29)$ & $8(11,4)$ & $0,001^{1}$ \\
\hline Ampicilina & $26(21)$ & $9(12,8)$ & $0,22^{1}$ \\
\hline Mortalidad a los 10 días de hospitalización & $3(2,4)$ & $1(1,4)$ & $0,10^{2}$ \\
\hline $\begin{array}{l}\text { Días de antibioticoterapia } \\
\text { (mediana, min-max) }\end{array}$ & 9 & 7 & $0,6^{3}$ \\
\hline${ }^{1} \chi^{2} .{ }^{2}$ Test exacto de Fisher. ${ }^{3}$ Mann Whitney. & $(2-60)$ & $(2-40)$ & \\
\hline
\end{tabular}

Con la introducción del check list en la UCIP se logró disminuir en $35 \%$ el escalamiento de antimicrobianos. Stocker y cols, implementando un check list, con el objetivo de mejorar la prescripción de antimicrobianos en la UCIP, incrementaron el uso apropiado de los antimicrobianos en cuadros infecciosos con hemocultivos negativos, de 18 a $74 \%(\mathrm{p}<0,001)^{10}$.

Con el check list la indicación de vancomicina en nuestro estudio se redujo en $25 \%$. Este resultado se consideró de interés, porque es uno de los antimicrobianos, cuya indicación ha ido incrementando en los pacientes críticos. Además de sus efectos de nefrotoxicidad, constituye un factor de riesgo de infección o colonización por microorganismos multi-resistentes como son Enterococcus spp resistentes a vancomicina y Klebsiella pneumoniae productora de $\beta$-lactamasas de espectro extendido (BLEE) $)^{11-14}$.

En el presente estudio, la intervención sobre la prescripción permitió documentar mejor la indicación de los antimicrobianos, al justificar el inicio del tratamiento y establecer un plan de tratamiento, basado en las posibilidades de infección del paciente, por manifestaciones clínicas o parámetros de laboratorio, ante la ausencia de hemocultivos positivos. En ambas fases del estudio, los retornos positivos de los hemocultivos fueron bajos; se atribuyó al hecho que un gran porcentaje de los pacientes ya se encontraba bajo tratamiento con antimicrobianos al ingreso a la UCIP. La toma de decisión ante un cultivo negativo en un paciente crítico es un desafío para el médico. Frecuentemente se consideran criterios de gravedad, estado general del paciente y microorganismos 
nos. El monitoreo de la prescripción de antimicrobianos sobre los médicos tratantes tiene el efecto de involucrarlos en forma directa en el uso apropiado, a través del mayor conocimiento de las especies bacterianas predominantes en la institución y la necesidad de trabajar en forma conjunta con infectólogos, laboratorio de microbiología y enfermeras ${ }^{19}$. De acuerdo a una revisión sistemática, las estrategias educacionales en los diferentes niveles de atención podrían ser las de mayor impacto ${ }^{20}$. La última revisión Cochrane, analizando las intervenciones realizadas en los hospitales con el objetivo de mejorar la prescripción de antimicrobianos, concluyó que son efectivas y seguras. La efectividad se evidencia por un mayor cumplimiento de los protocolos de administración y por la disminución de la duración la antibioterapia y la seguridad en la disminución de indicaciones innecesarias ${ }^{21}$.

El presente estudio presenta limitaciones por ser un estudio observacional realizado en un solo hospital. La inclusión, tanto de las fichas como de los pacientes en las dos etapas del estudio no fue aleatorizado. La etapa retrospectiva se realizó mediante la revisión manual de las historias clínicas porque no se cuenta con el formato electrónico. Tampoco se ha evaluado si la terapia empírica inicial fue apropiada porque se focalizó en el elevado porcentaje de escalamiento de antimicrobianos, a las $48 \mathrm{~h}$ observado en la cohorte histórica. Durante el lapso de tiempo entre el análisis retrospectivo y la introducción del check list no hubo cambios en el staff de la UCIP y las características de los pacientes. en ambos períodos fueron comparables.

Este estudio puede constituirse en el punto de partida para un estudio clínico randomizado utilizando el check list en la UCIP, con un mayor número de participantes y con un enfoque multidisciplinario, para la evaluación de su efectividad sobre la prescripción de los antimicrobianos.

\section{Conclusiones}

El check list aplicado en la UCIP donde se desarrolló el estudio disminuyó el escalamiento de antimicrobianos y la prescripción de vancomicina, sin aumentar la mortalidad a los 10 días de hospitalización. Además, permitió un mayor porcentaje de interconsultas con infectólogos. 
Anexo. Check list uso antibióticos UCIP

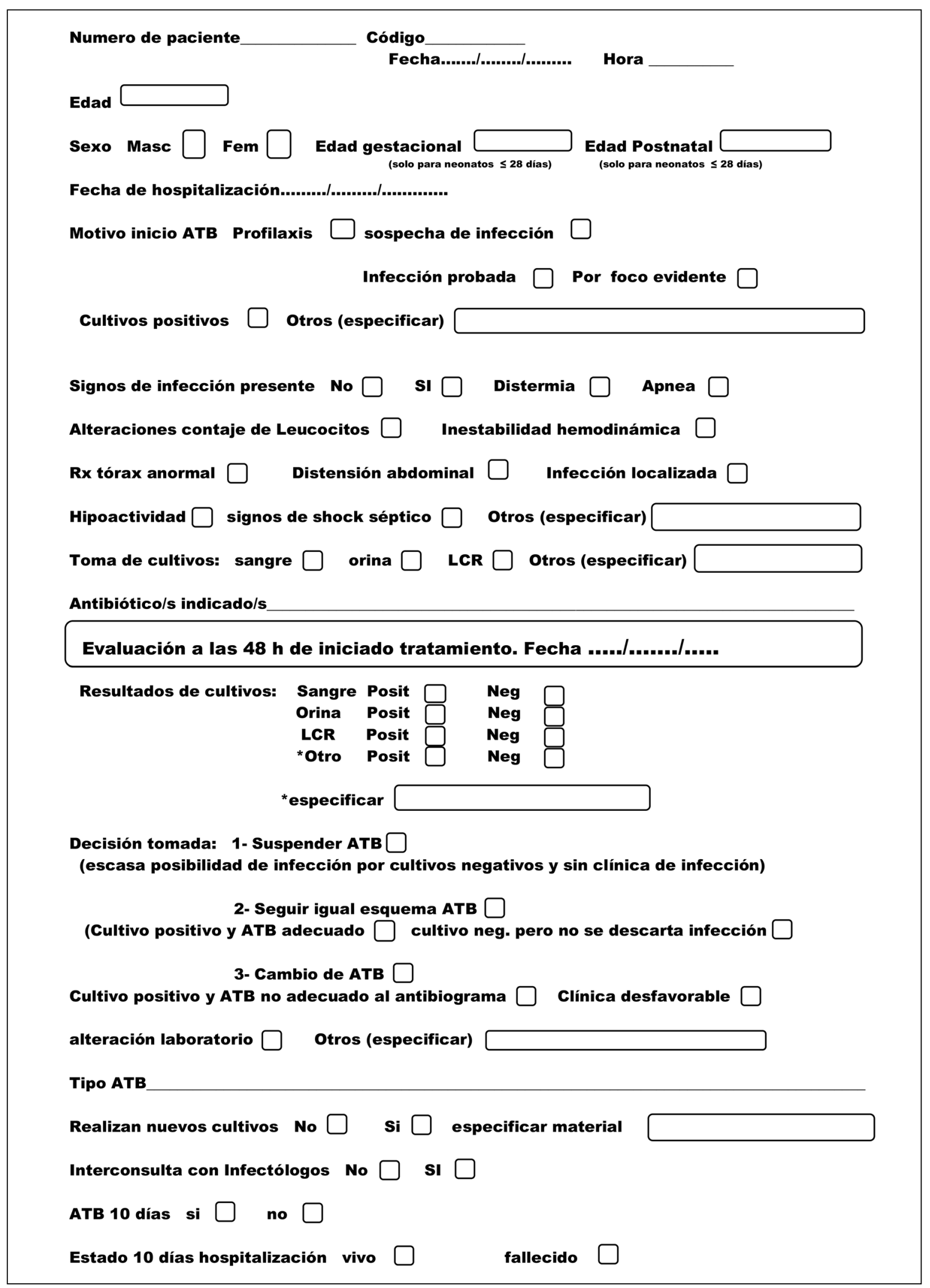




\section{Referencia s bibliográficas}

1.- Abbas Q, U1 Haq A, Kumar R, Ali S, Hussain $\mathrm{K}$, Shakoor S. Evaluation of antibiotic use in Pediatric Intensive Care Unit of a developing country. Indian J Crit Care Med. 2016; 20 (5): 291. doi: 10.4103/0972-5229.182197.

2.- Bidone N, Giglio N, Bakir J, Sheehan M G, Arias López M P, Rosin M, et al. Prescription and use of antibiotics at a pediatric intensive care unit in Buenos Aires City. Arch Argent Pediatr. 2008; 106(5): 409-15. doi: 10.1590/ S0325-00752008000500007.

3.- Vather A, Chb M B, Mckerrow N H, Sa F, Paed M, Morgan M E, et al. Factors underlying the changing of antibiotics in a paediatric intensive care unit in Pietermaritzburg , KwaZulu-Natal 2018; 12 (4). doi: 10.7196/SAJCH.2018. v12i3.1466.

4.- Downes K J, Weiss S L, Gerber J S, Klieger S B, Fitzgerald J C, Balamuth F, et al. A pragmatic biomarker-driven algorithm to guide antibiotic use in the pediatric intensive care unit: The optimizing antibiotic strategies in sepsis (OASIS) study. J Pediatric Infect Dis Soc 2017; 6 (2): 134-41. doi: 10.1093/jpids/piw023.

5.- Rhodes A, Evans L E, Alhazzani W, Levy M M, Antonelli M, Ferrer R, et al. Surviving sepsis campaign: International Guidelines for Management of Sepsis and Septic Shock: 2016. , Intensive Care Med 2017; 43: 304-77. doi: 10.1007/s00134-017-4683-6.

6.- Erku D A, Mekuria A B, Belachew S A. Inappropriate use of antibiotics among communities of Gondar town, Ethiopia: A threat to the development of antimicrobial resistance. Antimicrob Resist Infect Control 2017; 6 (1): 1-8. doi: 10.1186/s13756-0170272-2.

7.- Mali N B, Deshpande S P, Tullu M S, Deshmukh C T, Gogtay N J, Thatte U M. A prospective antibacterial utilization study in Pediatric Intensive Care Unit of a tertiary referral center. Indian J Crit Care Med 2018;
22: 422-6. doi: 10.4103/ijccm.IJCCM_365_17.

8.- Principi N, Esposito S. Antimicrobial stewardship in paediatrics. BMC Infect Dis [Internet] 2016; 16 (1): 1-8. doi: 10.1186/ s12879-016-1772-z.

9.- Dyar O J, Tebano G, Pulcini C. Managing responsible antimicrobial use: perspectives across the healthcare system. Clin Microbiol Infect [Internet] 2017; 23 (7): 441-7. doi: 10.1016/j.cmi.2017.04.016.

10.- Stocker M, Ferrao E, Banya W, Cheong J, Macrae D, Furck A. Antibiotic surveillance on a paediatric intensive care unit: Easy attainable strategy at low costs and resources. BMC Pediatr 2012; 12 (1):196. doi: 10.1186/14712431-12-196.

11.- Villena R, González CA, Nalegach ME, Vásquez A, Villareal M, Drago M. Monitoreo terapéutico de vancomicina intravenosa en una unidad de paciente crítico pediátrico. Rev Chilena Infectol 2014; 31 (3): 249-53. Disponible en http://repositorio.uchile.cl/ handle/2250/129587.

12.- Carmona F, Prado S I, Silva M F I, Gaspar G G, Bellissimo-Rodrigues F, Martínez R, et al. Vancomycin-resistant enterococcus outbreak in a pediatric intensive care unit: Report of successful interventions for control and prevention. Braz J Med Biol Res 2012; 45 (2): 158-62. doi: 10.1590/S0100-879X2012007500005.

13.- Somily A M, Alsubaie S S, Binsaeed AA, Torchyan A A, Alzamil F A, Al-Aska A I, et al. Extended-spectrum $\beta$-lactamase-producing Klebsiella pneumoniae in the neonatal intensive care unit: Does vancomycin play a role? Am J Infect Control 2014; 42 (3): 277-82. doi: 10.1016/j.ajic.2013.09.028.

14.- Flokas M E, Karageorgos S A, Detsis M, Alevizakos M, Mylonakis E. Vancomycinresistant enterococci colonisation, risk factors and risk for infection among hospitalised paediatric patients: a systematic review and meta-analysis. Int J Antimicrob Agents
2017; 49 (5): 565-72. doi: 10.1016/j. ijantimicag.2017.01.008.

15.- Lee TC, Frenette C, Jayaraman D, Green L, Pilote L. Antibiotic selfstewardship: Trainee-led structured antibiotic time-outs to improve antimicrobial use. Ann Intern Med. 2014; 161: S53-8. doi:10.7326/ M13-3016.

16.- Patel S J, Oshodi A, Prasad P, Delamora P, Larson E, Zaoutis T, et al. Antibiotic use in neonatal intensive care units and adherence with centers for disease control and prevention 12 step campaign to prevent antimicrobial resistance. Pediatr Infect Dis J 2009; 28 (12): 1047-51. doi :10.1097/INF.0b013e3181b12484.

17.- Bhullar H S, Shaikh F A R, Deepak R, Poddutoor P K, Chirla D. Antimicrobial justification form for restricting antibiotic use in a pediatric intensive care unit. Indian Pediatr 2016; 53 (4): 304-6. doi: 10.1007/s13312-0160841-0.

18.- Pulcini C, Botelho-Nevers E, Dyar OJ, Harbarth $\mathrm{S}$. The impact of infectious disease specialists on antibiotic prescribing in hospitals Clin Microbiol Infect [Internet]. 2014; 20 (10): 963-72. doi: 10.1111/1469-0691.12751.

19.- Hamilton K W, Gerber J S, Moehring R, Anderson D J, Calderwood M S, Han J H, et al. Point-of-prescription interventions to improve antimicrobial stewardship. Clin Infect Dis 2015; 60 (8): 1252-8. doi: 10.1093/cid/ civ 018

20.- Roque F, Herdeiro M T, Soares S, Rodrigues A T, Breitenfeld L, Figueiras A. Educational interventions to improve prescription and dispensing of antibiotics: A systematic review. BMC Public Health 2014; 14: 1276 https://doi. org/10.1186/1471-2458-14-1276.

21.- Charani E, Gould I M, Scott C L, Marwick C A, Ramsay C R, Davey P, et al. Interventions to improve antibiotic prescribing practices for hospital inpatients. Cochrane Database Syst Rev 2017; (2). CD003543. doi: 10.1002/14651858.CD003543.pub4. 